This item was submitted to Loughborough's Research Repository by the author.

Items in Figshare are protected by copyright, with all rights reserved, unless otherwise indicated.

\title{
Parallel array of YBa2Cu3O7- $\delta$ superconducting Josephson vortex-flow transistors with high current gains
}

\section{PLEASE CITE THE PUBLISHED VERSION}

http://dx.doi.org/10.1063/1.4819461

\section{PUBLISHER}

(c) AIP Publishing LLC

\section{VERSION}

VoR (Version of Record)

\section{LICENCE}

CC BY-NC-ND 4.0

\section{REPOSITORY RECORD}

Chesca, Boris, Daniel John, Matthew Kemp, Jeffrey Brown, and Christopher Mellor. 2019. "Parallel Array of Yba2cu3o7- $\delta$ Superconducting Josephson Vortex-flow Transistors with High Current Gains". figshare. https://hdl.handle.net/2134/13056. 


\section{AIP Appled Physics \\ Letters}

\section{Parallel array of YBa2Cu3O7- $\delta$ superconducting Josephson vortex-flow}

transistors with high current gains

Boris Chesca, Daniel John, Matthew Kemp, Jeffrey Brown, and Christopher Mellor

Citation: Appl. Phys. Lett. 103, 092601 (2013); doi: 10.1063/1.4819461

View online: http://dx.doi.org/10.1063/1.4819461

View Table of Contents: http://apl.aip.org/resource/1/APPLAB/v103/i9

Published by the AIP Publishing LLC.

\section{Additional information on Appl. Phys. Lett.}

Journal Homepage: http://apl.aip.org/

Journal Information: http://apl.aip.org/about/about_the_journal

Top downloads: http://apl.aip.org/features/most_downloaded

Information for Authors: http://apl.aip.org/authors

\section{ADVERTISEMENT}
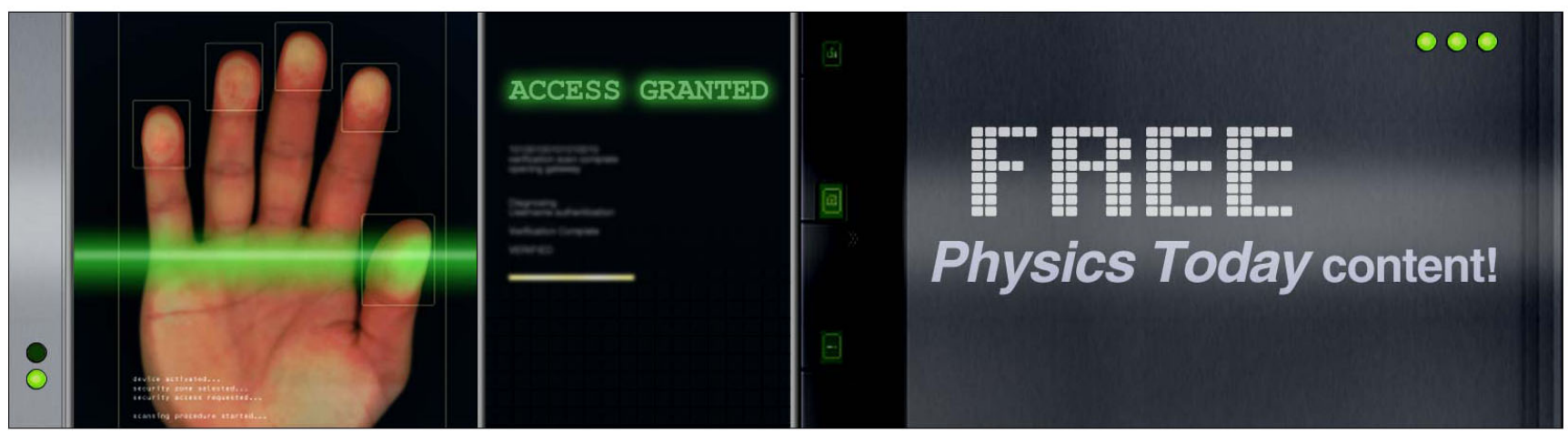


\title{
Parallel array of $\mathrm{YBa}_{2} \mathrm{Cu}_{3} \mathrm{O}_{7-\delta}$ superconducting Josephson vortex-flow transistors with high current gains
}

\author{
Boris Chesca, ${ }^{1}$ Daniel John, ${ }^{1}$ Matthew Kemp,${ }^{1}$ Jeffrey Brown, ${ }^{1}$ and Christopher Mellor ${ }^{2}$ \\ ${ }^{1}$ Department of Physics, Loughborough University, Loughborough, United Kingdom \\ ${ }^{2}$ School of Physics and Astronomy, Nottingham University, Nottingham, United Kingdom
}

(Received 21 June 2013; accepted 14 August 2013; published online 28 August 2013)

\begin{abstract}
We have developed a Josephson vortex-flow transistor based on a parallel array of 440 $\mathrm{YBa}_{2} \mathrm{Cu}_{3} \mathrm{O}_{7-\delta}$ bicrystal grain boundary Josephson junctions. The array's critical current $I_{c}$ was measured as a function of the control current $I_{c t r l}$ through a control line that is inductively coupled to the array. The device has a highly asymmetric $I_{c}\left(I_{c t r l}\right)$ curve with several regions where a switching behaviour is observed characterized by a maximum current gain $g_{\text {max }}=\partial I_{c} / \partial I_{\text {ctrl }}$ of 19 and a significant dynamic range of $20 \mu \mathrm{A}$ at $77 \mathrm{~K}$. In the range $4.7-92 \mathrm{~K} g_{\max }$ versus temperature is non-monotonic with a maximum recorded at 77 K. C 2013 AIP Publishing LLC.

[http://dx.doi.org/10.1063/1.4819461]
\end{abstract}

High temperature superconductor Josephson vortex-flow transistors (JVFTs) are the most promising candidates for high-frequency amplifying devices in all-superconducting microwave circuits. ${ }^{1,2}$ There are two types of JVFTs: ones that are based on a single long Josephson junction $(J J)^{3,4}$ or JVFTs based on arrays of $J J_{s} .^{5-7}$ A high performance JVFT for practical applications should have a high current gain, $g=\partial I_{c} / \partial I_{c t r l}$ (where $I_{c}$ is the device critical current and $I_{c t r l}$ is the control-gate current), a large dynamic range (the range of $I_{c t r l}$ over which a high $g$ can be achieved), small vortex transit times to allow high frequency operation and a relatively large transresistance, $r_{m}=\partial V / \partial I_{c t r l}$. Several JVFT designs have been proposed so far which differ in (i) current bias distribution (symmetric overlap ${ }^{1,3}$ or asymmetric inline $^{5-7}$ ), or (ii) gate line geometry (different gate design ${ }^{4}$ or different gate line relative orientation with respect to the junctions ${ }^{6}$ ). It has been shown ${ }^{1,3}$ that discrete JVTFs with an asymmetric current bias distribution have much larger current gains than discrete JVFTs with a symmetric one. This is due to self-field effects that translate into a highly asymmetric $I_{c}\left(I_{c t r l}\right)$ pattern. However, one significant drawback is that they are also characterized by relatively large vortex transit times and a small dynamic range in comparison to their symmetrical counterparts. ${ }^{8}$ In this paper we propose a discrete JVFT based on a parallel array of $440 \mathrm{YBa}_{2} \mathrm{Cu}_{3} \mathrm{O}_{7-\delta}$ bicrystal grain boundary $J J$ that combines beneficial aspects of several previous designs. On the one hand, it has a symmetric bias configuration to ensure a large dynamic range, as well as, small vortex transit times; on the other hand it has a certain degree of inductive asymmetry implemented via an asymmetric loop configuration within the array. This asymmetry ensures the bias current produces a net inhomogeneous magnetic field along the array through self-field effects that result in a highly asymmetric $I_{c}\left(I_{c t r l}\right)$ curve with high current gains. Of particular interest are JVFTs operating at $77 \mathrm{~K}$ due to their low-cost cooling procedures and practicality. There have been several reports of JVFTs with current gains $g$ as high as 6 at $30 \mathrm{~K}^{4}$ or 5.5 at $50 \mathrm{~K},{ }^{6}$ or 14 at $70 \mathrm{~K},{ }^{5}$ however, in all these cases $g(T)$ rapidly and monotonically decreases with temperature as it approaches $77 \mathrm{~K}$. Thus, the highest current gains reported so far at $77 \mathrm{~K}$ were achieved with discrete JVFTs having an asymmetric in-line bias distribution (maximum reported values were $\mathrm{g}=3.5^{7}$ ). Our discrete JVFT shows current gains as high as 19, a significant dynamic range of more than $20 \mu \mathrm{A}$ at $77 \mathrm{~K}$, and an unusual non-monotonic $g(T)$ dependence in the range $(4.7-92) \mathrm{K}$ with a maximum recorded value at $77 \mathrm{~K}$.

The JVFTs were fabricated by depositing high quality epitaxial, $100 \mathrm{~nm}$ thick $c$-axis oriented $\mathrm{YBa}_{2} \mathrm{Cu}_{3} \mathrm{O}_{7-\delta}$ (YBCO) films on $10 \times 10 \mathrm{~mm}^{2}, 24^{\circ}$ symmetric [001] tilt, $\mathrm{SrTiO}_{3}$ bicrystals by pulsed laser deposition. ${ }^{9}$ A $200 \mathrm{~nm}$ thick Au layer was deposited in situ on top of the YBCO film to facilitate fabrication of high quality electrical contacts for electric transport measurements. The films with a critical temperature, $T_{c}$, of $92 \mathrm{~K}$ were subsequently patterned by optical lithography and etched by an Ar ion beam to form a parallel array of $440 \mathrm{JJ}$. The array consists of 22 identical sets of $20 J J$ each. All $440 \mathrm{JJ}$ are $3 \mu \mathrm{m}$ wide. The junctions are separated by superconducting loops of identical width $(3 \mu \mathrm{m})$ but variable length. Thus, within each set of $20 J J$ the length of the loops varies logarithmically from $13 \mu \mathrm{m}$ to $8 \mu \mathrm{m}$. Consequently, the screening parameter $\beta_{L i}=2 \pi L_{i} I_{C J} / \Phi_{0}$ is also changing monotonically within each set of 19 superconducting loops. Here $\Phi_{0}$ is the flux quantum, $I_{c J}$ is the maximum Josephson critical current of each junction and $L_{i}$ is the inductance of the superconducting loop between $J J_{\mathrm{i}}$ and $J J_{\mathrm{i}+1}$, with $\mathrm{i}=1,2, \ldots, 19$. An optical micrograph of a small part of such a JVFT (containing 4 such sets of $20 J J$ each) is shown in Fig. 1. We designed our $J J$ array following several considerations as explained below. Previous investigations ${ }^{6,7}$ showed that for symmetric discrete JVTF with $\mathrm{N}=9,10$ and a constant value of $\beta_{L}$ throughout the array $\mathrm{g}\left(\beta_{L}\right)$ increases monotonically with $\beta_{L}$ and saturates for $\beta_{L}$ values larger than about 7. From numerical simulations of $I_{c}\left(I_{c t r l}\right)$ versus $\beta_{L}$ performed for asymmetric discrete $\mathrm{JVFT}^{1}$ it is found that $\mathrm{g}\left(\beta_{L}\right)$ saturates much faster, i.e., for $\beta_{L}$ values larger than about 1.5 for $\mathrm{N}=10$. Consequently, we designed our JJ array to have an average $\beta_{L}$ of about 1.5 at $77 \mathrm{~K}$. To ensure a highly asymmetric $I_{c}\left(I_{c t r l}\right)$ pattern with the high $\mathrm{g}$ values associated with it, we also implemented an asymmetric loop configuration 


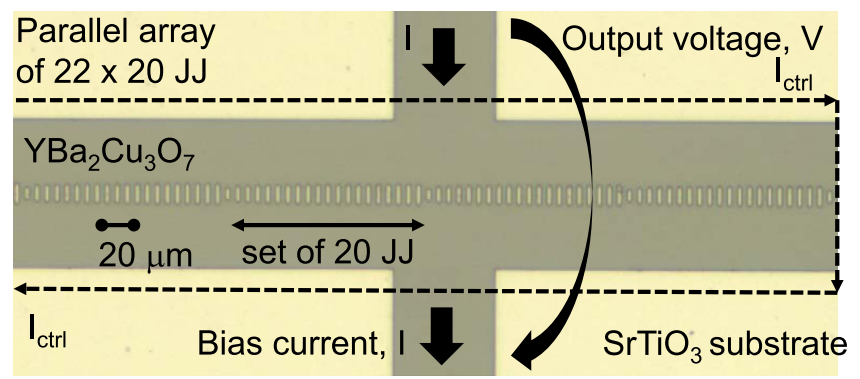

FIG. 1. Optical micrograph of the JVFT made of a parallel array of 22 identical sets of $20 \mathrm{JJ}$. Shown is a small central part of the array consisting of 4 sets of $20 \mathrm{JJ}$. Within each set all $20 \mathrm{JJ}$ are $3 \mu \mathrm{m}$ wide and are connected via superconducting loops of identical width $(3 \mu \mathrm{m})$ and variable length (logarithmically decreasing from $18 \mu \mathrm{m}$ to $13 \mu \mathrm{m}$ ). This geometry introduces an asymmetry with respect to the direction of the current bias $I$ and consequently an inhomogeneous net magnetic flux proportional to $I$ is produced along the array.

within the array by varying the value of $\beta_{L}$ monotonically within each set of $20 \mathrm{JJs}$ by about $\pm 15 \%$ around its average. We chose a logarithmic dependence for $\beta_{L}$ because it introduces a smaller degree of asymmetry than a linear dependence. The bias current, $I$, is applied symmetrically via the central top and bottom electrodes. The control current is fed through a control line (dotted line in Fig. 1), which is electrically decoupled from, and inductively coupled to, the array. We have fabricated two such devices and both showed a similar behaviour. In particular, the devices show a similar robust operation with or without a magnetically and electrically shielded environment which demonstrates the practicality of the design.

Families of $d c$ current-voltage $(I V)$ characteristics were measured by a 4 point-contact method at various temperatures between $4.7 \mathrm{~K}$ and $92 \mathrm{~K}$ and for different values of the control current $I_{c t r l}$ in the range $(-8 \mathrm{~mA}, 8 \mathrm{~mA}) . I_{c t r l}$ was changed in small steps of $15 \mu \mathrm{A}$. A family of 35 consecutive IVs measured at $77 \mathrm{~K}$ are plotted in Fig. 2. A maximum voltage modulation of $14 \mu \mathrm{V}$, due to a change in $I_{c t r l}$ between the first $I V$ ( $I V$ number 1 on Fig. 2) and the last $I V$ ( $I V$ number 4$)$ in this particular family, was recorded at a fixed current bias of $1 \mathrm{~mA}$. In blue are the two $I V$ 's numbered 2 and 3 for $I_{c t r l}$ range where a switching behaviour is observed: a small current of $15 \mu \mathrm{A}$ applied to the JVFT's gate increases the JVFT's drain-source current by $280 \mu \mathrm{A}$. That translates into current gains $g$ as high as 19. From such families of $I V$ ' $s$ scanned over $I_{c t r l}, I_{c}\left(I_{c t r l}\right)$ dependencies could be constructed for various voltage criteria (see Figs. 3 and 4) and a maximum value $g_{\text {max }}=\max \left(\partial I_{c} / \partial I_{c t r l}\right)$ for the current gain can be calculated. Remarkably, in contrast to previous reports on other types of discrete JVFTs, ${ }^{1-7} g_{\max }(T)$ is highly nonmonotonic with a maximum recorded value at $77 \mathrm{~K}$ (see inset of Fig. 2). From the measured $I V s$ in a magnetically shielded environment for each individual junction, $I_{c J}$ of about $3 \mu \mathrm{A}$ and $I_{C J} R_{N}$ product of about $50 \mu \mathrm{V}$ were calculated at $77 \mathrm{~K}$ (where $R_{N}$ is the junction's normal resistance). That translates into a Josephson critical current density $J_{c}$ of about $1 \times 10^{3} \mathrm{~A} / \mathrm{cm}^{2}$. As expected by using $J J$ bicrystal technology for the implementation of our concept only relatively modest values for its transresistance, $r_{m}$, of about $0.04 \Omega$ at $77 \mathrm{~K}$ were achieved.

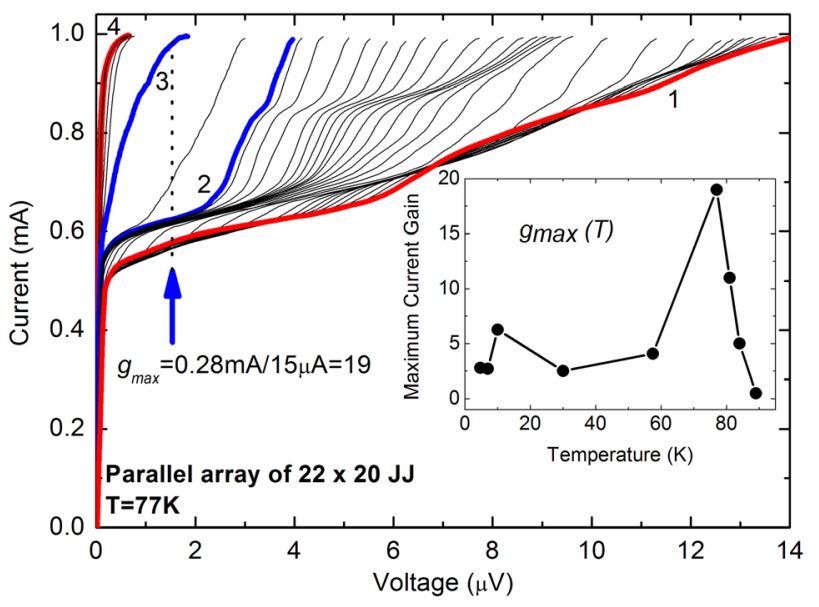

FIG. 2. A set of 35 consecutive current-voltage $I V$ characteristics recorded at $77 \mathrm{~K}$ for various values of the control current $I_{\text {ctrl }}$ around $1.2 \mathrm{~mA}$. $I_{\text {ctrl }}$ is changed in steps of $15 \mu \mathrm{A}$. In red are the two $I V \mathrm{~s}$ numbered 1 and 4 for the extreme values of $I_{c t r l}$. In blue are the two $I V$ s numbered 2 and 3 for the $I_{c t r l}$ range where a switching behaviour is observed with current gains as high as 19. Inset shows a very unusual maximum current gain $g_{\max }(T)$ dependence in the range $(4.7,89) \mathrm{K}$ with a maximum recorded value at $77 \mathrm{~K}$.

At temperatures close to $T_{c}$ we are in the low-inductance limit in which $\beta_{L i}$ are negligibly small and the magnetic fields of the bias current $I$ can be neglected. Therefore, the magnetic field dependence of the array critical current, $I_{c}$, is unaffected by $I$ and should have a symmetric and periodic pattern consisting of a series of maxima similar to a diffraction pattern of an optical grating consisting of multiple slits. ${ }^{10}$ The periodicity in $I_{\text {ctrl }}$ corresponds to one additional flux quantum $\Phi_{0}$ in each loop. This is confirmed by the experiments as shown in Fig. 3 where $I_{c}\left(I_{c t r l}\right)$ for 5 different voltage criteria between $-0.2 \mu \mathrm{V}$ and $-1 \mu \mathrm{V}$ measured at $89 \mathrm{~K}$ show a symmetric and periodic pattern with a periodicity of about $2 \mathrm{~mA}$. We performed numerical simulations based on a model developed earlier ${ }^{10}$ in the limit of small $\beta_{L}$ and from the modulation of $I_{c}$ with $I_{c t r l}$ we estimate an average value for $\beta_{L}$ of 0.3 at $89 \mathrm{~K}$. As the temperature is decreased $\beta_{L i}$ can no longer be neglected (due to an increase in $I_{C J}$ ) and consequently the self-field effects of the supercurrents become important. Since the inductance values of individual loops change monotonically within each set of 19 consecutive loops, due to differences in their lengths, a significant asymmetry appears between the left and the right half of the device as far as the self-induced flux in the loops is concerned. As a result, the bias current $I$ produces a net inhomogeneous magnetic flux along the array that is proportional to $I$. The additional flux due to the self-field effects causes an increased (positive feedback) and a reduced slope (negative feedback) of the increasing and decreasing branches, respectively, of the $I_{c}\left(I_{c t r l}\right)$ curve measured at $89 \mathrm{~K}$. This is because the same change of the applied flux $\Phi_{c t r l}$ (produced by $I_{c t r l}$ ) results in larger or smaller changes of the critical current of the device. An asymmetric $I_{c}\left(I_{c t r l}\right)$ curve is therefore expected for this device at $T<89 \mathrm{~K}$ along with the associated high current gains. The asymmetry of $I_{c}\left(I_{c t r l}\right)$ as well as $g_{\text {max }}$ are expected to increase rapidly with decreasing temperature as self-field effects (that are proportional to $\beta_{L i}$ ) are enhanced according to the temperature dependence of $I_{C J}$. Such a behaviour is indeed observed in a set 


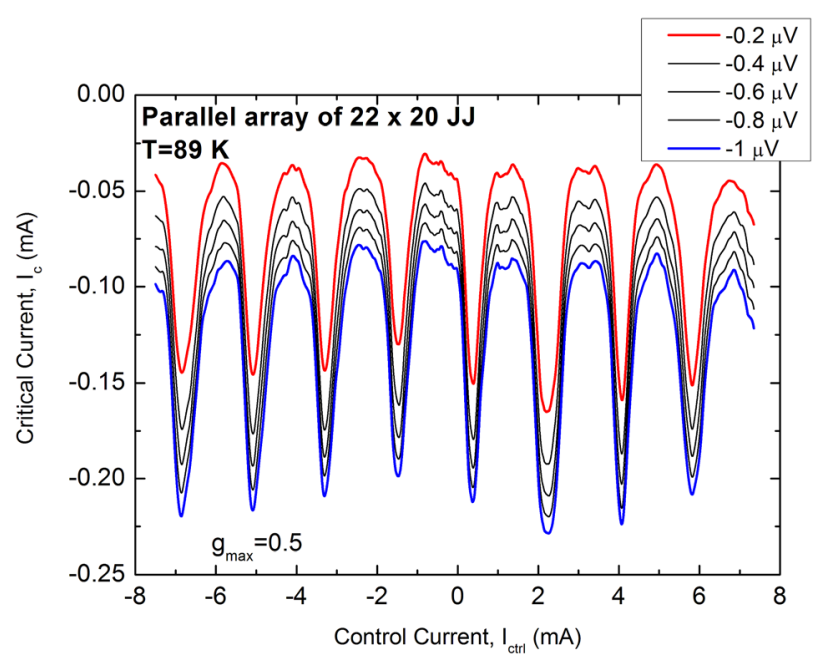

FIG. 3. $I_{c}\left(I_{c t r l}\right)$ at $89 \mathrm{~K}$ for 5 different voltage criteria between $(-1,-0.2) \mu \mathrm{V}$ showing a symmetric and periodic pattern with a periodicity of about $2 \mathrm{~mA}$.

of measurements taken at $89 \mathrm{~K}, 84 \mathrm{~K}, 81 \mathrm{~K}$, and $77 \mathrm{~K}$ (see Figs. 4(a) and 4(b)) where the degree of $I_{c}\left(I_{c t r l}\right)$ asymmetry and $g_{\text {max }}$ both gradually increase as we decrease $T$. A similar rapid transition with increasing $\beta_{L}$ (decreasing temperature) from a symmetric $I_{c}\left(I_{c t r l}\right)$ to an asymmetric one was predicted to occur in discrete JVFTs with an asymmetric current bias distribution. ${ }^{1,7,8}$ Considering the increase in $I_{c}$ due to a change in temperature from $89 \mathrm{~K}$ to $77 \mathrm{~K}$ an average value for $\beta_{L}$ of 1.9 can be estimated at $77 \mathrm{~K}$ which is close to the designed value of 1.5 . In contrast to $I_{c}\left(I_{c t r l}\right)$ curves, $V\left(I_{c t r l}\right)$ curves are almost symmetric at all temperatures.

A switching behaviour is observed at $77 \mathrm{~K}$ with current gains as high as $g=19$ and a corresponding dynamic range of $\Delta I_{\text {ctrl }}>20 \mu \mathrm{A}$ for positive voltages (see Fig. 4(a)) and $g=10$ with a corresponding $\Delta I_{c t r l}>15 \mu \mathrm{A}$ for negative voltages (Fig. 4(b)). A similar behaviour is observed at $81 \mathrm{~K}$ as well, with slightly smaller values for $g$ and $\Delta I_{c t r l}$ associated with it. Interestingly, a switching behaviour that is due to strong self-field effects with very high values of $g$ associated with it have been predicted theoretically for JVFTs with an asymmetric bias current distribution. ${ }^{1,8}$ However, it has never been observed experimentally. In our case the switching behaviour has a different nature, as explained below. First, it should be noted that flux-flow resonance modes occur in our system that are due to the motion of a chain of vortices through the discrete $J J$ array, accompanied by an emission of small amplitude linear waves that propagate along the array. When the vortex spacing is commensurate with the wavelength of the emitted waves flux-flow resonances are produced on the family of $I V{ }^{\prime} s{ }^{11,12}$ Two such resonances, which are tunable in a magnetic field (i.e., their voltage location strongly depends on the applied magnetic field via $I_{c t r l}$ ), are clearly visible in Fig. 2. A detailed investigation of these resonances will be published elsewhere. ${ }^{13} \mathrm{~A}$ breakdown of these flux-flow resonance modes occurs within a very narrow range of $I_{c t r l}$ (that corresponds to the two blue $I V s$ numbered 2 and 3 on Fig. 2). This triggers a sharp transition involving several hundred $J J_{S}$ from a state where the array's $I_{c}$ has a value close to a minimacorresponding to destructive interference of individual $\left(I_{C J}\right)_{\mathrm{i}}$
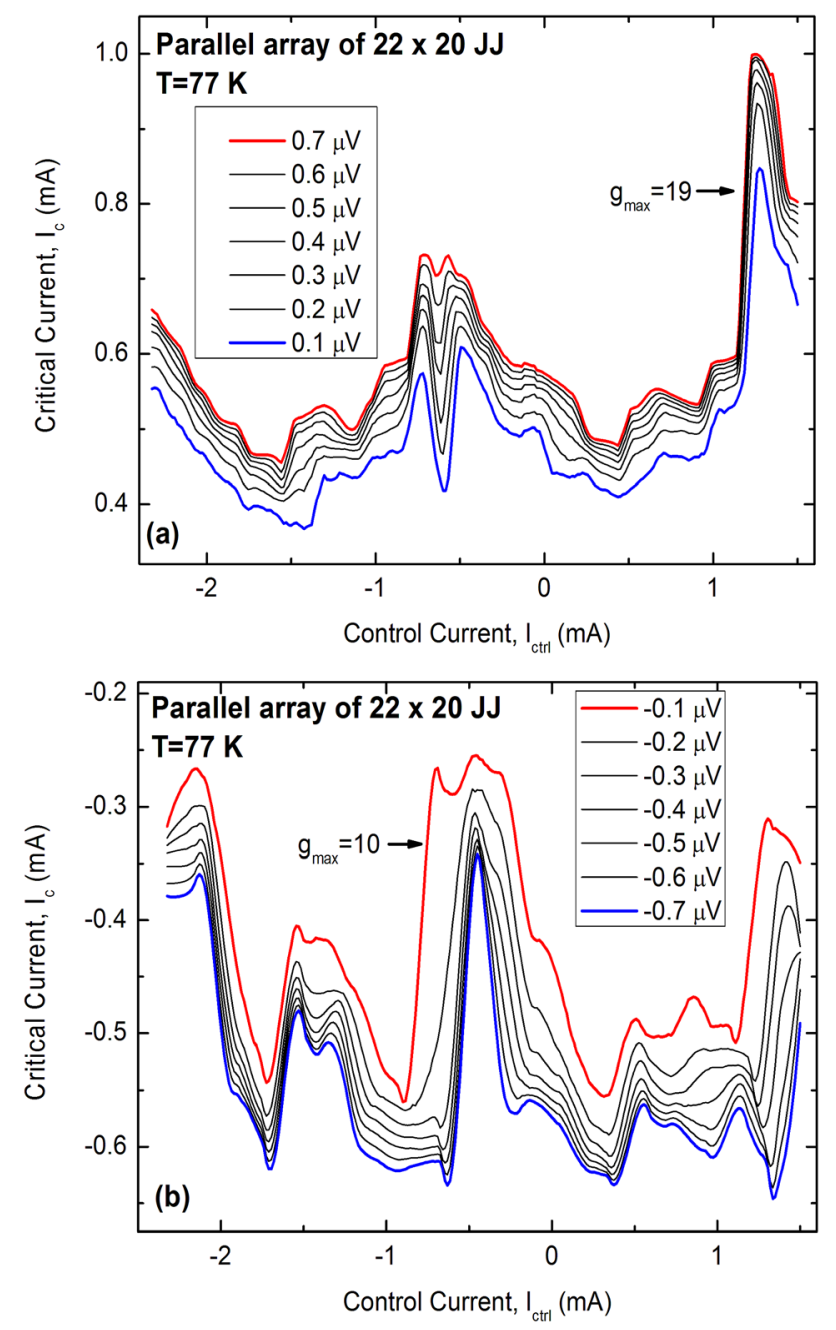

FIG. 4. (a) $I_{c}\left(I_{c t r l}\right)$ at $77 \mathrm{~K}$ for 7 different voltage criteria between $(0.1,0.7)$ $\mu \mathrm{V}$ showing a highly asymmetrical pattern. A switching behaviour is clearly visible at about $I_{c t r l}=1.2 \mathrm{~mA}$ with currents gains as high as 19 and a dynamic range of more than $20 \mu \mathrm{A}$. (b) Similar to (a) except for voltage criteria between $(-0.7,-0.1) \mu \mathrm{V}$. Again a switching behaviour is clearly visible at about $I_{c t r l}=-0.8 \mathrm{~mA}$ with currents gains as high as 10 and a dynamic range of more than $15 \mu \mathrm{A}$.

(see blue $I V$ numbered 2 on Fig. 2) - to a state where the array's $I_{c}$ is close to its maxima - corresponding to constructive interference of individual $\left(I_{C J}\right)_{\mathrm{i}}$ (see the blue $I V$ numbered 3 on Fig. 2).

It is important to note that at lower temperatures $(4.7 \mathrm{~K}$, $10 \mathrm{~K}, 30 \mathrm{~K}$, and $57.5 \mathrm{~K}) g_{\max }$ values are significantly smaller (see inset of Fig. 2). Although never observed experimentally such a non-monotonic $g(T)$ dependence characterized by a maximum has been predicted by numerical simulations performed for discrete JVFT's with an asymmetric current bias distribution. ${ }^{7}$ In our case the physics behind this can be understood as follows: decreasing the temperature leads to a decrease in the losses experienced by moving fluxons (i.e., smaller damping) accompanied by an increased discreteness of the array (the discreteness parameter is $\left(\beta_{L}\right)^{1 / 2}$ ). At some threshold temperature, $T_{t h}$, numerical simulations show that flux-flow resonances are completely suppressed and a transition to a chaotic regime occurs. ${ }^{12}$ Consequently, in our case for temperature below $T_{t h}$ (which appears to be around $77 \mathrm{~K}$ ) 
the switching behaviour and the high gains associated with the flux-flow resonances both vanish.

We have developed a JVFT prototype based on arrays of $J J$ with an asymmetric loop configuration and demonstrated its operation in a wide temperature range 4.7-92 K. The device shows very promising performance in the areas of current gain and dynamic range. For some applications a primary concern might be the low impedance, $r_{m}$, of these devices. This issue can be solved by using an alternative to $J J$ bicrystal technology (e.g., a ramp-type $J J$ ) for the implementation of our concept. The device operates in both magnetically and electrically unshielded environments and at $77 \mathrm{~K}$ demonstrating its potential for practical devices. The design approach reported here shows great promise as a route to realizing high performance supercomputing amplifiers using JVFTs.

The authors would like to thank Dominic Walliman and Jas Chauhan for their technical support.
${ }^{1}$ R. Gross, R. Gerdemann, L. Alff, T. Bauch, A. Beck, O. M. Froehlich, D. Koelle, and A. Marx, Appl. Supercond. 3, 443 (1995).

${ }^{2}$ J. Mannhart, Supercond. Sci. Technol. 9, 49 (1996).

${ }^{3}$ Y. M. Zhang, D. Winkler, P. A. Nilsson, and T. Claeson, Appl. Phys. Lett. 64, 1153 (1994).

${ }^{4}$ S. J. Berkowitz, Y. M. Zhang, W. H. Mallison, K. Char, E. Terzioglu, and M. R. Beasley, Appl. Phys. Lett. 69, 3257 (1996).

${ }^{5}$ R. Gerdemann, T. Bauch, O. M. Froehlich, L. Alff, A. Beck, D. Koelle, and R. Gross, Appl. Phys. Lett. 67, 1010 (1995).

${ }^{6}$ J. Schuler, S. Weiss, T. Bauch, A. Marx, D. Koelle, and R. Gross, Appl. Phys. Lett. 78, 1095 (2001).

${ }^{7}$ T. Bauch, S. Weis, H. Haensel, A. Marx, D. Koelle, and R. Gross, IEEE Trans. Appl. Supercond. 7, 3605 (1997).

${ }^{8}$ B. A. Davidson, V. Granata, E. Sarnelli, and S. Pagano, Supercond. Sci. Technol. 12, 970 (1999).

${ }^{9}$ The films were deposited by Theva $\mathrm{GmbH}$.

${ }^{10}$ J. H. Miller, G. H. Gunaratne, J. Huang, and T. D. Golding, Appl. Phys. Lett. 59, 3330 (1991).

${ }^{11}$ H. S. J. van der Zant, T. P. Orlando, S. Watanabe, and S. H. Strogatz, Phys. Rev. Lett. 74, 174 (1995).

${ }^{12}$ A. V. Ustinov, M. Cirillo, B. H. Larson, V. A. Oboznov, P. Carrelli, and G. Rotoli, Phys. Rev. B 51, 3081 (1995).

${ }^{13}$ B. Chesca, D. John, and C. J. Mellor, "Amplification of electromagnetic waves excited by a chain of propagating vortices in YBCO Josephsonjunction arrays" (unpublished). 